\title{
A Xeno-Free, Serum-Free Expansion Medium for Ex-vivo Expansion and Maintenance of Major Human Tissue-Derived Mesenchymal Stromal Cells
}

\author{
Lopes Vanda S*, Annie Ngo and Hsiao-Tzu Ni
}

Department of Research and Development, Irvine Scientific Inc, Santa Ana, CA, USA

*Corresponding author: Lopes VS, Department of Research and Development, Irvine Scientific Inc., Santa Ana, CA, USA, Tel: +1 949-261-7800; E-mail: vlopes@irvinesci.com

Rec date: Mar 19, 2018; Acc date: May 03, 2018; Pub date: May 7, 2018

Citation: Vanda SL, Ngo A, Tzu Ni H (2018) A Xeno-Free, Serum-Free Expansion Medium for Ex-vivo Expansion and Maintenance of Major Human Tissue-Derived Mesenchymal Stromal Cells. Transl Biomed. Vol.9 No.2:146

\section{Abstract}

Introduction: MSCs, one of the most useful stem cell type for clinical applications, can be derived from various adult tissues. While sharing the same basic properties, there are however differences in their proliferative capacity, degree of potency and level of multipotent marker expression and immunosuppression ability. As the cell media industry continues to develop effective serum-free cell culture media, an understanding of the potential effect on MSCs profile and functionality is needed.

Methods and Findings: MSCs derived from adipose tissue, bone marrow and umbilical cord were grown in FBS containing media, or a newly-developed XSFM and characterized by flow cytometry, immunomodulation and differentiation assays. In this study we have found that each source of MSCs had a different cell expansion profile, but cells grown in XSFM reach higher cumulative cell numbers. No significant difference was noted in the expression profile of CD45, CD105 and CD90 and cells retained their differentiation and their immunomodulatory potential. BM-derived MCSs had a relatively weaker immunomodulatory potential, but this could be improved when cells were cultured in XSFM.

Conclusion: In conclusion, the developed XSFM for human MSCs provides a superior alternative for clinical applications with multiple cell origins, by eliminating the drawbacks of sera usage, while leading to higher cumulative cell numbers and maintaining the cell characteristics.

Keywords: Mesenchymal stem/stromal cells; Xeno-free; Serum-free; Culture media

\section{Introduction}

40 plus years of research on mesenchymal stromal cells (also named as mesenchymal stem cells, MSCs), combined with the discovery of their ability to support hematopoiesis, ability to differentiate along mesenchymal and non- mesenchymal lineages, and to have immunomodulation properties has led human MSCs to become one of the most clinically used stem cell type [1-5]. MSCs were first identified in bone marrow by Friedenstein et al. in 1974 [6] and were subsequently discovered and derived from other postnatal tissues, including adipose, dental pulp, umbilical cord, cord blood, placenta and amniotic fluid [7-12]. Although all of these different human tissue- derived cells exhibit the basic properties that allowed them to be classified as MSCs [13], they also present some differences in their proliferative capacity, degree of potency and level of multipotent marker expression [14-18].

More than 360 clinical trials employing human MSCs have been initiated, targeting various diseases. The combination of their non-immunogenic profile with anti-inflammatory properties reduces the potential for any side effect, such as fibrosis or tissue rejection. The list of clinical applications includes the use of bone marrow derived MSCs (BM-MSCs) for Crohn's disease, myocardial infarction, spinal cord injury and osteoarthritis; adipose tissue derived MSCs (AD- MSCs) for multiple sclerosis and spinal cord injury; umbilical cord derived MSCs (UC-MSCs) for type I diabetic and ulcerative colitis [19], among others. On average, $10^{7}-10^{8}$ cells per kg per dose are required for clinical treatments, but MSCs have a limited longterm expansion, making it a challenge to generate the high cell numbers required for these therapeutic dosages. Similarly, to what happens in most conventional cell culture conditions, the majority of these trials involve MSCs expanded in bovine serum containing media.

In 2014, FDA reported that out of 66 MSCs drug applications, $80 \%$ used FBS during their manufacturing [20]. Bovine serum- containing media appear to support the needed quantitative cell expansion and be able to maintain MSCs cell characteristics, with no major adverse reactions described so far. However, the undefined nature and biological origin of bovine serum comes with the potential risk of transmission of unknown zoonotic agents and raises concerns in the ability to generate consistent quality-control cells due to batch-to-batch variation. It has been estimated that with a dosage of $10^{8}$ cells expanded in bovine serum containing media $7 \mathrm{mg}$ to $30 \mathrm{mg}$ of bovine proteins are infused [21], which in turn can lead to immunological responses [22]. The use of bovine serum creates also the need to add a wash step prior to cell infusion, 
but even in that case it seems that the internalized xenogeneic antigens cannot be removed [23]. Finally, it also raises animal welfare concerns due to the number of animals used. All these issues combined with the slow, yet steady, decline in the quality of the bovine serum supply [24] further stresses the necessity to develop an effective serum-free cell culture media for biological drug production in biopharmaceutical industry settings, including MSCs-based therapies. Alternatives to the use of animal derived components have been tested, such as allogeneic and/or autologous human platelet lysate (HPL) preparations [25]. However, these do not avoid the variability between batches, undefined composition, method of large scale production and the safety issues.

MSCs-based therapies are considered as Advanced Therapy Medicinal Products (ATMPs). The focus for clinical applications of MSCs is now on a safe, reproducible large scale and highquality production of cells. Previous reports have focused on studying other commercially available XSFM but were limited to either analysing one specific cell source or did not determine the effect of the media on immunomodulation properties [25-35]. Here we report the development of a xenofree, serum-free expansion medium (PRIME-XV ${ }^{\circledast}$ MSCs Expansion XSFM, also named XSFM throughout the manuscript) for adipose tissue-, bone marrow- and umbilical cord- derived human MSCs that can generate high cell numbers and maintain the cell characteristics, including immunomodulation properties. XSFM expansion media can be a superior alternative to expand and culture various major tissue-derived human MSCs, applicable from the bench to a large-scale platform, while maintaining their cell phenotypes, multipotency and immunomodulation potential.

\section{Methods}

\section{Cell culture}

Three different primary human MSCs (adipose tissue-, bone marrow-, and umbilical cord-derived) were purchased from ATCC (Manassas, VA, USA) and Lonza (Walkersville, MD, USA). A master cell bank was generated at passage $(P)$ by culturing the MSCs in PRIME- XV ${ }^{\circledast}$ MSCs Expansion XSFM (Irvine Scientific, Catalog \#91149, CA, USA) and freezing the MSCs with PRIME-XV ${ }^{\oplus}$ MSCs FreezIS DMSO-Free (Irvine Scientific, Catalog \#91140, CA, USA) freezing media. The MSCs used in all experimental assays were derived from the P2 master cell banks, thawed and seeded in T75-flasks pre-coated with fibronectin (Prime-XV $\mathrm{V}^{\circledast}$ Human Fibronectin, Irvine Scientific, CA, USA), at a density of $6.0 \times 10^{3}$ cells $/ \mathrm{cm}^{2}$ in serumcontaining $\alpha$-MEM (10\% qualified FBS lot) (Irvine Scientific, Catalog \#9144, CA, USA) or PRIME-XV ${ }^{\circledast}$ MSCs Expansion XSFM (Irvine Scientific, Catalog \#91149, CA, USA). MSCs were fed every 2-3 days and passaged with TrypLE Select (Thermo Fisher Scientific, CA, USA) when a confluency of $70 \%$ to $80 \%$ was reached. Viable cell number and viability was measured using the Vicell hematology analyzer (Beckman Coulter, CA, USA). Doubling times were calculated from three independent experiments using the formula DT (doubling time) $=\mathrm{t} \log 2 /$ $[(\log C f)-(\log C i)]$, where $t$ is time in hours, $C f$ is the final cell number and $\mathrm{Ci}$ the initial cell number plated.

\section{Flow cytometry analysis}

Cell marker expression was assessed by flow cytometry analysis of cell surface protein expression, using a BD FACSVerse flow cytometer (BD Biosciences, CA, USA). $1 \times 10^{5}$ cells were stained with APC anti-human CD90 (Biolegend, Clone 5E10, CA, USA), PE anti-human CD105 (Biolegend, Clone 43A3, CA, USA), and PerCP/Cy5.5 anti-human CD45 (Biolegend, Clone $\mathrm{HI} 30, \mathrm{CA}, \mathrm{USA})$ at $4^{\circ} \mathrm{C}$ for 30 minutes, in the dark. To set background fluorescence levels, unstained and matched isotype controls were used. For isotype controls, we used APC Mouse IgG1, PE Mouse IgG1, and PerCP/Cy5.5 Mouse IgG1 (all Biolegend, Clone MOPC-21, CA, USA). Percentage of positive cells for each marker was calculated from three independent experiments.

\section{Mesenchymal lineage differentiation assay}

The Mesenchymal lineage differentiation potential was determined by culturing the MSCs for 2 to 4 weeks in PRIME$X^{\circledast}$ Adipogenic SFM (Irvine Scientific, Catalog \#91137, CA, USA), PRIME-XV ${ }^{\circledast}$ Osteogenic Differentiation SFM (Irvine Scientific, Catalog \#91132, CA, USA), or PRIME-XV ${ }^{\circledast}$ Chondrogenic Differentiation XSFM (Irvine Scientific, Catalog \#91138, CA, USA) prior to staining. For adipogenic differentiation, MSCs were cultured in four well plates (Nunc, ThermoFisher Scientific, USA) at a seeding density of 3.2-3.7 $\times$ $10^{4}$ cells per well in either serum-containing $\alpha$-MEM or in PRIME-XV ${ }^{\circledast}$ MSCs Expansion XSFM. When the MSCs reached $70 \%-80 \%$ confluency (1-2 days post seeding), the media was exchanged to PRIME-XV ${ }^{\circledast}$ Adipogenic SFM (Irvine Scientific, CA, USA). The MSCs were then cultured for an additional 8-10 days, with media exchanges at every 2-3 days. On day 7, adipose vacuoles were noticeable in the culture. MSCs were stained on day 10 with goat anti-mouse FABP4 (R\&D Systems, Catalog \#AF1443, USA) and DAPI (ThermoFisher Scientific, Catalog \#D1306, USA). For osteogenic differentiation, MSCs were cultured in four well plates (Nunc, ThermoFisher Scientific) at a seeding density of $7.4 \times 10^{3}$ to $1 \times 10^{4}$ cells per well in either serum-containing $\alpha$ - MEM or in PRIME-XV ${ }^{\oplus}$ MSCs Expansion XSFM.

When the MSCs were $50 \%$ to $70 \%$ confluent (2- 3 days post seeding), the media was switched to PRIME-XV ${ }^{\circledR}$ Osteogenic Differentiation SFM (Irvine Scientific, Catalog \#91132, CA, USA). The MSCs were cultured for an additional 10-14 days with a media exchange at every 2-3 days. MSCs were stained on day 14 with mouse anti- human Osteocalcin (R\&D Systems, Clone 190125, USA). For chondrogenic differentiation, $2.5 \times$ $10^{5}$ cells were spun down in a $15 \mathrm{ml}$ falcon tube and cultured in PRIME-XV ${ }^{\circledast}$ Chondrogenic Differentiation XSFM (Irvine Scientific, Catalog \#91138, CA, USA). Within 24 hours, a chondroblast spheroid should form at the bottom of the falcon tube. The MSCs were cultured for an additional 28 days, with a media changes every 2-3 days. On day 28 , the chondroblast spheroid was embedded and cryosectioned. Sections were stained with mouse anti-human Collagen type II (R\&D Systems, 
Catalog \#AF361, USA), goat anti-human Aggrecan (R\&D Systems, Catalog \#AF1220, USA), and DAPI (ThermoFisher Scientific, Catalog \#D1306, USA), and imaged under the same settings. Images were analyzed and fluorescence intensity was determined using ImageJ software.

\section{Immunomodulation assay}

Immunomodulation assay was established in 6-well tissue culture plates with a total volume of $2 \mathrm{ml}$ per well. MSCs and $\mathrm{CD}^{+} \mathrm{T}$ cells (Astarte Biologics, USA) as well as anti-CD3 and anti- CD28 antibodies (clones UCHT1 and CD28.2, respectively, Biolegend, CA, USA), were all prepared in PRIME-XV ${ }^{\circledast}$ MSC Expansion XSFM. Preparation of MSCs for this assay included a wash and resuspension at $6 \times 10^{4}$ cells per well in $1 \mathrm{ml}$ of medium. $\mathrm{CD}^{+} \mathrm{T}$ cells were labelled with $1 \mu \mathrm{L}$ CellTrace ${ }^{\mathrm{TM}}$ CFSE (carboxyfluorescein diacetate succimidyl ester, ThermoFisher Scientific, USA) per $1 \times 10^{6}$ cells, for 7 minutes at $37^{\circ} \mathrm{C}$ in the dark. CFSE labeling of $\mathrm{T}$ cells allows us to measure $\mathrm{T}$ cell proliferation, with the intensity of CFSE decreasing with each round of cell division. At the end of incubation, five times the labeled volume was added to stop the CFSE labeling reaction. A final concentration of $3 \times 10^{5} \mathrm{CFSE}$-labeled $\mathrm{CD}^{+} \mathrm{T}$ cells per well in $2 \mathrm{ml}$ of medium was added into the appropriate conditions. To establish a reliable gating strategy, we used a non-stimulated control consisting of a ratio of $5: 1 \mathrm{CD}^{+} \mathrm{T}$ :MSCs cells without the addition of anti-CD3 and anti-CD28. T cells in these non-stimulated control wells did not proliferate, yet their survival was maintained by the presence of MSCs. In no instance did MSCs induce T-cell proliferation without the addition of both anti-CD3 and anti-CD28 antibodies, which demonstrates the robustness of the negative control. In addition, we also used stimulated control consisting of $\mathrm{CD}^{+} \mathrm{T}$ cells with the addition of anti-CD3 and anti-CD28 antibodies at a final concentration of $1 \mu \mathrm{g} / \mathrm{ml}$. T cells in this stimulated control proliferated at the end of incubation. A MSCs only control was also included. For the immunomodulation assay, a ratio of $5: 1 \mathrm{CD}^{+} \mathrm{T}$ to MSCs, with both anti-CD3 and anti-CD28 antibodies at a final concentration of $1 \mu \mathrm{g} / \mathrm{ml}$, were seeded in a final volume of $2 \mathrm{ml}$. Cells were cultured for 4 days at $37^{\circ} \mathrm{C}$. The proliferation profile of $\mathrm{T}$ cells was analyzed by flow cytometry. Anti-human CD4 antibody conjugate was used to gate $\mathrm{CD}^{+}$cells. All flow cytometry analysis was performed using FACSVerse (BD Biosciences, USA).

\section{Statistical Analysis}

Analysis was performed by using a Student's two sample unpaired $t$ test.

\section{Results}

Development of a xeno-free, serum-free MSCs expansion media through a rational design process

The Rational Culture Media Design ${ }^{\circledR}$ is a media development platform built upon Irvine Scientific's cell culture experience and is a quality driven approach where measured and more rational steps are taken from beginning to end in developing new media. The platform consists of three steps carried out sequentially (Figure 1a).

The first step is the Design input and is where the desired functionality and properties of the medium are determined. For the development of a XSFM the criteria include: the medium is suitable for translational studies; supports expansion of multi-tissue derived MSCs while maintaining potency; it is ready-to use (without the need to add additional supplements) and contains no animal derived components (XF). The second step is Quality by Design. At this step specific problems are analysed via hypothesis testing, rather than trying multiple variables and determining what works. This is largely accomplished by making use of our cell culture history combined with a Design of Experiment methodology, which allows us to examine the subtle differences in component concentration and the synergistic/antagonistic effect of each component. Material qualification is actively considered during this process, to ensure the quality and consistency of each component. The third step is Verification, where the ultimate goal is to ensure that the Design inputs were addressed and that the media developed is robust and performs consistently.

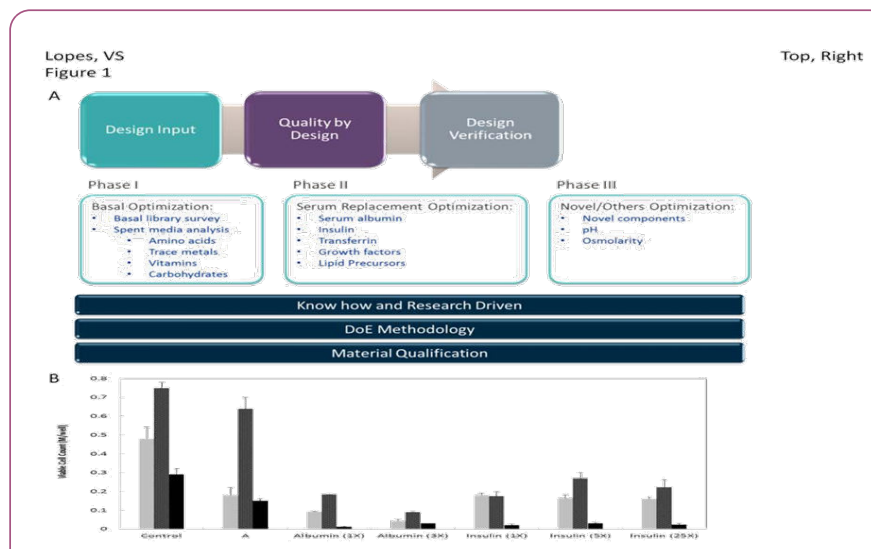

Figure 1 Rational culture media design process. (a) Diagram of a Rational Culture Media Design Process and step-wise approach taken during the development of serum, xenofree medium. (b) Representative example of a rational designed experiment designed to replace serum components by looking at albumin and insulin versus an existing control prototype (labeled as control) and a leading supplier's medium (labeled as $A$ ), $n=3$.

The Quality by Design step can be broken down into three phases (Figure 1a). For XSFM development, the process started with a basal medium optimization, where an intensive survey of basal medium is applied along with a spent media analysis. This allowed a more in-depth understanding of the various metabolic changes during MSCs culture and to determine the need for supplementation of basic components in a medium (e.g., salts, carbohydrates, and amino acids). The next phase evaluated serum replacement options. For XSFM development, the focus is on the replacement of all animalderived components with human-derived counterparts, without affecting functionality of the medium. 
Here we considered the known alternatives to bovine serum albumin (BSA). Figure $\mathbf{1 b}$ shows an example of a rational designed experiment where replacement options, including albumin and insulin, were tested along with the effects of varying the amount of the replacement component. The last phase evaluates components that are not self-evident. These may include lipids/lipid derivatives, modified amino acids, $\mathrm{pH}$ and osmolality. By following this design platform, we developed a ready to use XSFM consisting of an optimized basal medium supplemented with serum, xeno-free alternatives (such as clinical grade human serum albumin), combined with an optimized serum-replacement protein/lipid mix (including insulin, transferrin and chemically-defined lipids).

\section{Ex-vivo growth of MSCs derived from adipose tissue, bone marrow, and umbilical cord in serum-containing media and XSFM}

Adipose tissue-, bone marrow-, and umbilical cord-derived MSCs were thawed in serum-containing media (10\% FBS in $\alpha$ MEM) or the XSFM formulation. Within 24 hours most of the cells had adhered to the culture vessel. AD- and UC -derived MSCs seemed to adhere quicker to the culture vessel quicker than BM-derived MSCs. The cells grown in serum-containing medium exhibited a more flattened, fibroblast-like morphology, independently of the source, all throughout their ex-vivo growth (Figure 2a). This flat morphology became more apparent with increasing passage number, particularly in the case of BM-derived MSCs. In the presence of XSFM, MSCs derived from the three tissues maintained their morphology and proliferative capability through three passages and exhibited a relatively more homogenous and distinct spindleshaped morphology (Figure 2b). The morphology was also comparable in all tissue derived MSCs throughout expansion (Figure 2b), contrary to what was observed in serum containing media.

To test the ability of serum-containing medium to support continued expansion of multi-tissue derived MSCs passage cells were expanded for three additional consecutive passages (Figure 2c). While serum-containing medium does support the continual expansion of MSCs there is small difference in growth rates between the three cell sources. We observed a greater rate of expansion in AD-derived MSCs, followed by UCand BM-derived MSCs. The viability was maintained throughout expansion, with a $>90 \%$ at the end of each expansion. XSFM was also able to supports the continual expansion of MSCs, with a similar trend in growth rates and viability (Figure $2 \mathbf{d}$ ). While both serum containing media and XSFM were able to support expansion of MSCs for 3 passages, a comparison of the cumulative cell numbers shows that cells grown in XSFM have a better expansion. This is further demonstrated by the representative population doubling times (Figure 2e). AD-MSCs expanded in XSFM had a faster doubling time, taking twice as much time in serum containing media. This difference can be higher in the case of BM-MSCs (Figure 2e), which can end up in a relevant time saving when one considers the need to generate enough cells in the minimal amount of time for therapy applications. Of note is also the variability seen when MSCs were grown in FBScontaining media, ranging from 40 to $109 \mathrm{hrs}$ depending on the cell source, and within it. Cells expanded in XSFM have similar doubling times, with very little variability (Figure 2e). A previous study with another serum-free media reached a similar result, concluding that in this case the PDT was more associated with the media used than the cell origin. However, that media was unable to support BM-MSCs [29].

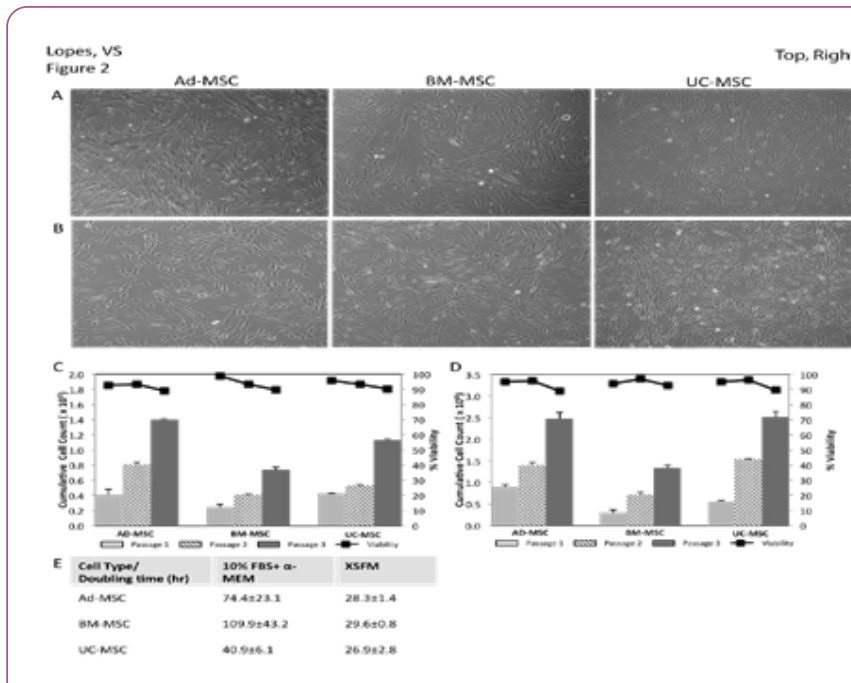

Figure 2 Morphology and growth curves of MSCs expanded in $10 \%$ FBS $+\alpha-M E M$ or XSFM. (a, b) Bright field images of adipose tissue-(AD-MSCs), bone marrow-(BM-MSCs), and umbilical cord-derived MSCs (UC-MSCs) were cultured on human fibronectin coated culture vessels in serumcontaining $10 \%$ FBS $+\alpha$-MEM (a) or XSFM (b), at a seeding density of 6,000 cells per $\mathrm{cm}^{2}$. Images were taken prior to passaging at $10 \mathrm{X}$ magnification. (c,d) Graphical representation of the cumulative cell number and viability of MSCs at different passages when expanded in serum containing media (c) or XSFM (d). Viability across the three passages is represented by the black line. Data shown represents $n=6$, and 3 different lots per cell source. (e) Doubling time, in hours, for AD-MSC, BM-MSC, and UCMSCs across three passages. The $p$ value is $\leq 0.05$ between cells grown in FBS containing media and XSFM, for all tissue sources. $n=3$.

\section{Immunophenotype of MSCs after ex-vivo expansion in XSFM}

To test the phenotypical characteristics of the expanded MSCs cell-surface antigen expression was analysed by flow cytometry for both positive and negative representative markers from MSCs. All MSCs expanded in serum-containing medium displayed comparable characteristic surface profiles, including positive expression of CD90 and CD105 (>95\%) and negative expression of CD45 (<1\%) (Figures 3a-3c). The small difference seen in the intensity of CD105 expression can be attributed to donor variability and/or the tissue source. The same was observed when MSCs were expanded in XSFM 
(Figures $\mathbf{3 b}$ and $\mathbf{3 c}$ ). Interestingly, when compared to the cells grown in serum-containing medium, cells expanded in XSFM seem to have lower levels of CD105, with AD-derived MSCs showing the greatest down-regulation of CD105. This observation was found consistently with multiple donors, and other reports have also demonstrated lower expression of CD105 for human MSCs cultured in serum-free medium versus a serum-containing counterpart [26].

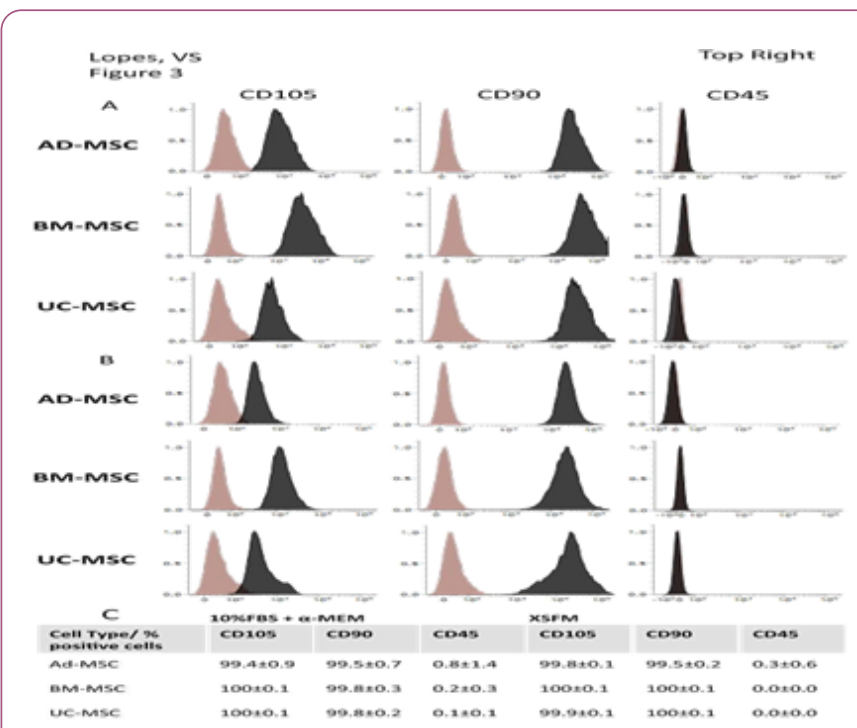

Figure 3 Immunophenotype of MScells expanded in two different medium. MSC-specific marker expression was analysed by flow cytometry. The graphs show representative profiles for CD105, CD90, and CD45 expression at the end of the third passage from all three tissue-derived MSCs (black) expanded in 10\% FBS (a) or xeno- and serum- free media. (b) Background fluorescence levels were set by using matched isotype controls (orange). (c) Percentage of positive cells expressing CD105, CD90 and CD45, determined by flow cytometry, $n=3$.

\section{Multipotentiality of MSC expanded under different media conditions}

To test the multi-lineage differentiation potential of AD-, BM-, and UC-MSCs we used a typical mesenchymal lineage differentiation protocol. As shown in Figure 4, AD- and BMderived MSCs grown for three passages in serum-containing media retained the ability to differentiate into adipocytes, osteoblast, and chondrocytes, as seen by the expected morphology and positive staining for FABP4, Osteocalcin, and Aggregan/Collagen Type II, respectively (Figures 4a-4c). Interestingly, we observed a limited potential of the BM- and UC-derived MSCs to differentiate into adipocytes, as seen by the lower number of FABP4 positive cells (Figures $\mathbf{4 a}$ and $\mathbf{4 g}$ ). These findings may indicate endogenous tissue source differences and may be of importance for the clinical application of human MSCs expanded in serum-containing medium.

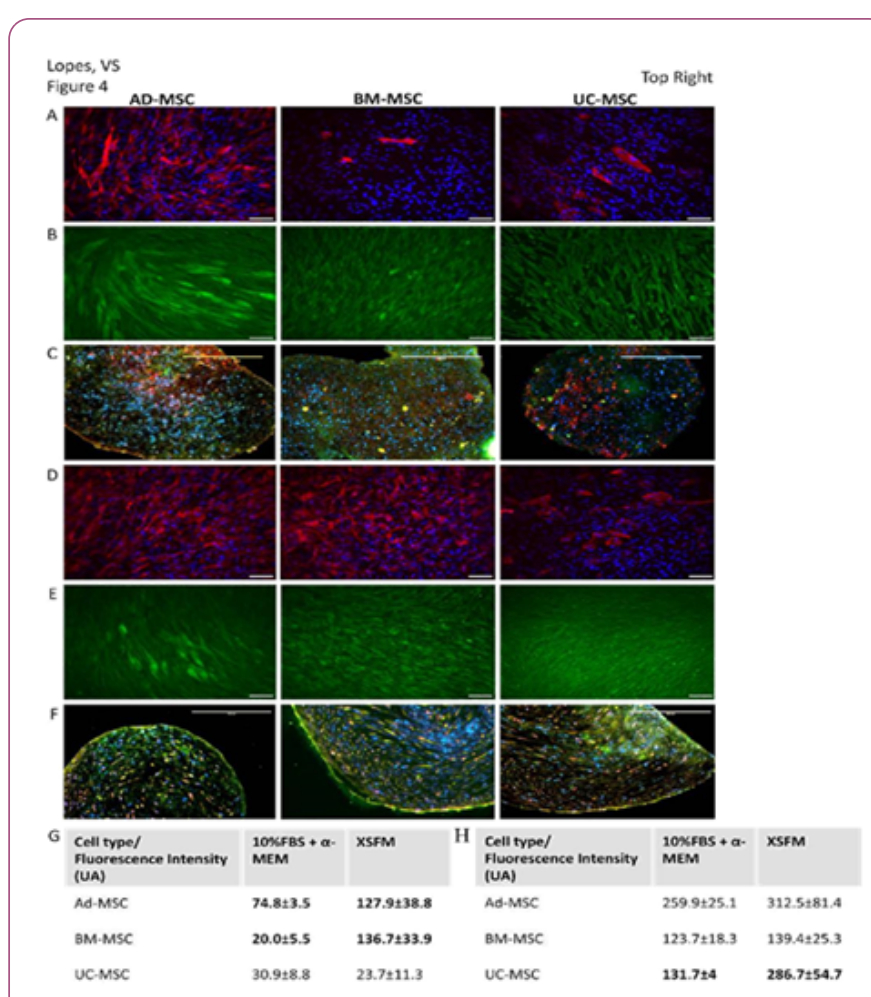

Figure 4 Mesenchymal-lineage differentiation of AD-MSC, BM-MSC, and UC-MSCs. All MSCs were expanded in serumcontaining medium (a-c) or XSFM (d-f) prior to differentiation. ( $a$, d) MSCs were cultured in PRIME-XV Adipogenic Differentiation Medium SF for 14 days and stained with FABP4 (red) and DAPI (blue). (b, e) MSCs were cultured in PRIME-XV Osteogenic Differentiation Medium SF for 14 days and stained for Osteocalcin (green). (c, f) MSCs were cultured in PRIME-XV Chondrogenic Differentiation Medium XSFM for 28 days prior to cryo-sectioning and staining with collagen type II (green), Aggrecan (red), and DAPI (blue). Scale bars for A, B, D, E=100 $\mu$; Scale bar for C, $\mathrm{F}=300 \mu \mathrm{m}$. $(\mathrm{g}, \mathrm{h})$ Fluorescence intensity quantifications for adipogenic (g) and osteogenic differentiation (h). Values in bold represent samples with $p \leq 0.05$. $n=3$.

In the case of MSCs expanded for three passages in XSFM, the tri-lineage differentiation could also be observed (Figures 4d-4f). AD-MSCs were able to differentiate into the three lineages efficiently, as in the case of cells expanded in serum containing media. UC-MSCs showed the same profile of differentiation as seen with cells grown in FBS containing media. However, BM -derived MSCs seem to efficiently differentiate into adipocytes, in contrast to what was observed with FBS-containing media (Figure $\mathbf{4 g}$ ).

A comparison between both FBS-containing and XSFM (Figures $\mathbf{4 g}$ and $\mathbf{4 h}$ ) indicates that the potency of the MSCs is impacted by the expansion medium, with XSFM resulting in an overall higher potency level. The other relevant factor that affects MSCs differentiation potential is the tissue source. 
Immunogenicity and Immunosuppressive potential of MSCs expanded in the presence of serum or XFSM.

To test the immuno-modulatory activity of different source derived MSCs, an immunomodulation co-culture protocol using anti-CD3 and anti-CD28 antibodies to stimulate CFSE labeled $\mathrm{CD}^{+} \mathrm{T}$ cells was used. Baseline controls were established by culturing CFSE labeled $\mathrm{CD}^{+}{ }^{+} \mathrm{T}$ cells alone $199.64 \%$ in non-proliferative state, $\mathrm{P} 1$, and $0.36 \%$ in proliferative state, $\mathrm{P} 2$ ), and CFSE labeled $\mathrm{CD}^{+} \mathrm{T}$ cells in the presence of anti-CD3 and anti-CD28 antibodies $(75.60 \%$ in proliferative state, P2) (Figure 5a). At a ratio of 5:1 $\left(\mathrm{CD}^{+} \mathrm{T}\right.$ cells to MSCs), suppression of $\mathrm{CD}^{+} \mathrm{T}$ cells was observed with all three tissue-derived MSCs when cultured in serumcontaining medium. Compared to the baseline control proliferation of $75.60 \%$, proliferation was measured to be $32.66 \%, 62.62 \%$, and $42.62 \%$ for AD-, BM-, and UC-derived MSCs, respectively (in the proliferative state, P2). BM-derived MSCs demonstrated a weaker immunosuppressive response compared to the other tissue-derived MSCs.

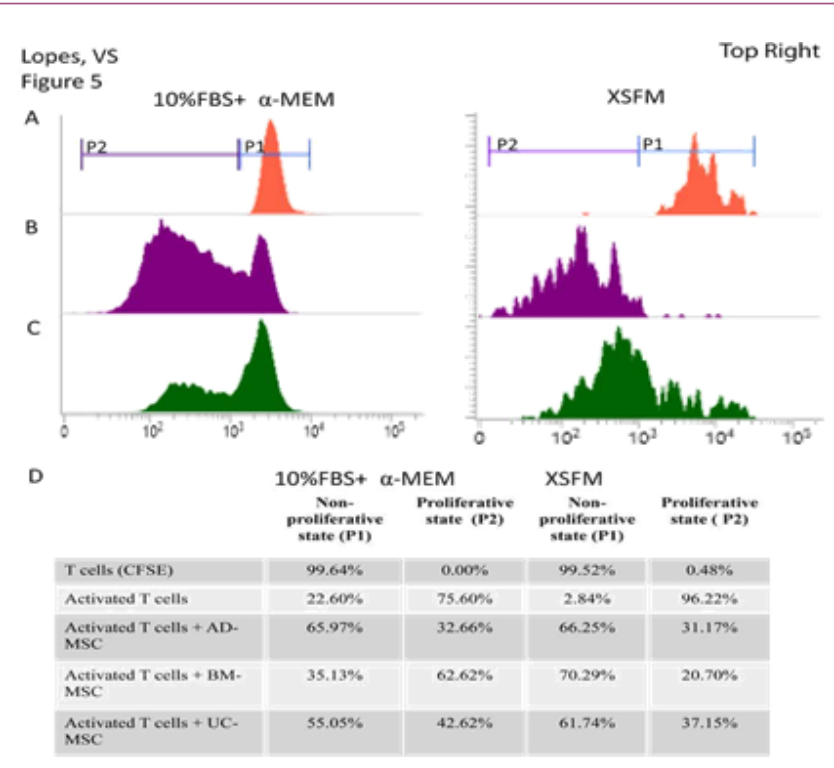

Figure 5 Immunomodulatory potential of AD-MSC, BMMSC, and UC-MSCs. CFSE levels were measured via flow cytometry to asses $\mathrm{CD}^{+} \mathrm{T}$ cell proliferation. AD-MSC flow results are shown as a representative. $(a, b)$ CFSE-labeled $\mathrm{CD}^{+} \mathrm{T}$ cells in the absence (a) Or presence (b) Anti-CD3 and anti-CD28 antibodies were cultured for controls. (c) Coculture of CFSE- labeled $\mathrm{CD}^{+} \mathrm{T}$ cell in the presence of antiCD3 and anti-CD28 antibodies and MSCs (green curve). (d) Summary of the different conditions tested and the determined T cell proliferation.

The same test was performed for MSC grown in XSFM. The baselines controls were $99.52 \%$ in the non-proliferative state, $\mathrm{P} 1$, and $0.48 \%$ in proliferative state $\mathrm{P} 2$ for non- activated $\mathrm{T}$ cells, and $96.22 \%$ in proliferative state, $\mathrm{P} 2$, for activated T cells (Figures $\mathbf{5 b}$-5d). At a ratio of 5:1 we observed a suppression of
$\mathrm{CD}^{+} \mathrm{T}$ cell proliferation to less than half in all three tissuederived MSCs (Figures $\mathbf{5 c}$ and $\mathbf{5 d}$ ).

Comparison between the two medium tested indicates that immunosuppression is greater with MSCs cultured in XSFM (Figure 5d). Interestingly, while BM-MSCs cultured in serumcontaining medium had the least suppressive effect (about $13 \%)$ of the three tissue-derived MSCs, it had the most improvement of their immunosuppressive effect (about 75\%) when grown in XSFM, becoming comparable to the other two tissue-derived MSCs. This is indicative of the intrinsic molecular differences that exist among different tissue-derived MSCs, and improvements to the potency of MSCs can be made through media development. However, the potential clinical effect may still vary based on the source of the MSCs.

\section{Discussion}

The use of MSCs in the clinical arena is a rapidly advancing field, with more than 360 ongoing clinical trials. It is thus becoming more pressing to find ways to reduce inherent variabilities involved in the process of generating MSCs, while still being able to get cells in adequate numbers and quality for human therapy. It is becoming mandatory to find a large-scale production method that is reproducible, and that allows a standard operating procedure (SOP) to be established. Variation in cell source, cell isolation methods and expansion conditions need to be addressed and ideally cells should be manufactured under GMP conditions. Expansion media has classically been composed of FBS, which carries critical safety issues: batch to batch variation, undefined composition, xenogeneic components and viral contamination possibility. The replacement of FBS with human platelet-rich plasma has been tested with success [2], but it does not alleviate the concerns related to biological contamination, scarcity of material and variability. Here we describe the use of PRIME$\mathrm{XV}^{\circledast}$ MSCs Expansion XSFM media, a cGMP product, versus serum containing media for expansion of three different sources of MSCs. While there are some previous reports that address the impact of using serum-free media in MSC culture, they have focused on either one single cell source [32-34] or have not studied the impact on immunomodulation profiles [36], a crucial aspect for MSCs application in the clinic.

In order to facilitate the transition from basic research to the clinical application of MSCs the successful development and introduction of a new media needs several factors to be considered. Firstly, while MSCs derived from various adult tissues have been shown to exhibit similar properties, which allowed them to be defined as MSCs, differences have also been noted (e.g., proliferative capacity, degree of potency, and level of multipotent marker expressions). UC- MSCs seemed to have a higher proliferative potential and also lack genome aberrations due to their postnatal origin. That combined with the fact that their source is considered a medical waste makes them an attractive source of MSCs. On the other hand, ADMSCs are abundant and present at any age. Thus, multi-tissue sourced MSCs were tested, to ensure a non-restrictive use of PRIME-XV ${ }^{\circledast}$ MSC Expansion XSFM media. 
Summarized in Table 1 are the results obtained with ADBM-, and UC-MSCs expanded in both serum-containing medium and the newly developed XSFM. We observed a more morphologically homogenous population of distinct spindleshaped morphology when MSCs were cultured in XSFM. This morphology was consistent through 3 passages. On the other hand, increased flattened edges were observed in MSCs cultured in serum-containing medium. This morphology difference has also been reported by others $[27,28]$ and it may be related to the adhesive properties of the cells. The adhesive properties of MSCs are influenced by the media used, with XF and SF media frequently requiring surface coating [27].

Table 1 Comparison of the results obtained from culturing AD-, BM-, and UC-MSCs in xeno- and serum-free expansion medium versus serum-containing medium.

\begin{tabular}{|c|c|c|c|c|c|c|}
\hline \multirow[b]{3}{*}{ Morphology } & \multicolumn{3}{|c|}{ Serum containing Media } & \multicolumn{3}{|c|}{ Xeno and serum-free media } \\
\hline & AD-MSC & BM-MSC & UC-MSC & $\begin{array}{l}\text { AD- } \\
\text { MSC }\end{array}$ & BM-MSC & UC-MSC \\
\hline & \multicolumn{3}{|c|}{ Fibroblast-like, some flattened edges } & \multicolumn{3}{|c|}{ Homogeneous, distinct spindle-shaped } \\
\hline Expansion & ++ & $+/-$ & + & +++ & + & ++ \\
\hline \multicolumn{7}{|c|}{ Immunophenotyping } \\
\hline CD105 & ++ & ++ & ++ & + & + & + \\
\hline CD90 & +++ & +++ & +++ & ++ & +++ & +++ \\
\hline CD45 & - & - & - & - & - & - \\
\hline \multicolumn{7}{|c|}{ Differentiation Potential } \\
\hline Adipogenic & ++ & + & + & ++ & ++ & + \\
\hline Osteogenic & + & + & + & + & + & + \\
\hline Chondrogenic & + & + & + & + & + & + \\
\hline Immunomodulation & +++ & ++ & +++ & +++ & +++ & +++ \\
\hline
\end{tabular}

An important parameter for a successful clinical application of MSCs is the dosage, and the ability to generate the number required from a small initial cell population, in a shorter and cost-effective form, without compromising the quality of the product. The removal of serum resulted in a quicker expansion of MSCs, with a reduction to half in the doubling time for ADMSCs, and even a bigger difference for bone marrow-derived cells. This indicates that the XSFM provides superior efficiency in MSC expansion, while requiring less medium, surface area and time, all of which are relevant aspects in large scale cell expansion. Similar results were obtained when another defined media formulation was compared to FBS and Allohuman serum containing preparations $[28,29]$. The in vitro replicative senescence has also been shown to be reduced when XF media is used [29], which contributes to the higher levels of proliferation and ability to reach higher passage numbers. Both characteristics are advantageous, by decreasing the manufacturing time and accelerating the production of cells in sufficient numbers. In addition, the use of XSFM also results in less variability, as seen in the doubling times. The decrease in variability between different tissue-sources and donors is highly relevant from a controlled process point of view. While using of FBS containing media will require the manufacturing process to be adjusted for every donor, it seems that a standard process can be established when using XSFM. However, the relative trend in the growth rate of the three tissue sources was similar in both mediums. AD-MSCs had the fastest rate of growth while BM-MSCs had the slowest rate. This supports the notion that the differences between sources seen can be attributed the endogenous differences of the cell origin, and not the media. Another XF media available in the market has been reported to support the growth of ADMSC, but not BM-MSCs [28], which seems to indicate that PRIME-XV ${ }^{\circledast}$ MSC Expansion XSFM may be a better suited media for multi-source MSC expansion.

In addition to cell expansion, it is also critical that cells maintain their characteristics. We have found the expected MSC marker expression profile, which was maintained throughout expansion. Consistent with previous reports [26,35], a down-regulated CD105 was observed when ADMSCs were cultured in XSFM. CD105 is a membrane glycoprotein and part of the transforming growth factor- $\beta$ (TGF- $\beta$ ) receptor complex. It has been shown that TGF- $\beta$ can upregulate CD105 transcription under hypoxic conditions [37] but the mechanism behind this influence, as well as its functional benefit, is unknown. CD105 is also particularly sensitive to enzymatic and cell damage by physical mechanisms, so it is possible that the lower adhesive properties of MSCs in XSFM make it more sensitive to be lost. A previous report has also found a decrease in CD54 and other minor differences in immune-related genes when comparing a XF-SF media versus FBS containing expansion media [27], but the effect of these changes is not clear, and further studies may be required to determine its clinical relevance. In our hands, MSCs from the three sources studies were able to differentiate into adipocytes, osteocytes and chondrocytes. We have found that XSFM increased the potential of AD- and 
BM-MCSs to differentiate into adipocytes, and osteogenic potential of UC-MSCs. The low adipogenic potential found in serum-containing media is an indication that this is not an optimal media for MSCs, as they seem to lose their ability to efficiently follow the trilineage differentiation. Other studies found that chondrogenic differentiation was potentiated in a XF $[27,37]$ while osteo- and adipogenic differentiation was decreased [27]. These discrepancies may be due to the lower cell adhesion described for the XF media used in that study, or media formulation.

Finally, we found that the MSCs were able to suppress CD3+ $T$ cell proliferation when cultured in both XSFM and serumcontaining medium. The degree of immunomodulation potential was found to be tissue source dependent, but also impacted by the media as a superior suppression in $\mathrm{CD}^{+} \mathrm{T}$ cell proliferation was found when MSCs were cultured in XSFM. Similarly, Swamynathan et al., [28] reported a slightly better immunomodulation response in XF media, although not statistically significant. Of interest is the fact no other study has done a comparison on immunomodulation profiles in multiple sources of MSCs side by side in serum-containing and XSFM media. These results suggest that MSCs characteristics can thus be impacted by the expansion medium and tissue source.

\section{Conclusion}

The development of a XSFM for the expansion of multitissue derived MSCs represents a necessary step in developing the adequate tools required to study human MSCs in a consistent and reproducible manner. An off the shelf product will have as minimal requirements that stem cell characteristics are maintained, have high proliferation rate and the cells to be functional. These requirements are met by PRIME-XV ${ }^{\circledast}$ MSC Expansion XSFM media. By eliminating the use of non-human derived components, such as fetal bovine serum and bovine serum albumin, this cGMP produced XSFM provides the ability for researchers from different laboratories and translational clinics to conduct studies with similar reagents and with reduced lot-to-lot variability. Use of XSFM usually requires surface coating for good cell adhesion, adding an extra step and cost. While PRIME-XV ${ }^{\circledast}$ MSC Expansion XSFM media benefits from the use of coating, studies indicate that it can still perform in its absence (data not shown). In conclusion, PRIME-XV ${ }^{\circledast}$ MSC Expansion XSFM, made with quality raw materials, can be used with several tissue sources of MSCs, while being able to generate higher numbers of cells and maintaining or improving the characteristics of MCSs. PRIME$\mathrm{XV}^{\oplus}$ MSC Expansion XSFM is thus a promising candidate for MSC clinical grade production of MSC in complete xeno-free conditions.

\section{Conflicts of Interests}

The author(s) declare that there is no conflict of interest regarding the publication of this article.

\section{Author Contributions}

Vanda S. Lopes: Conception and design, collection and/or assembly of data, data analysis and interpretation, manuscript writing Annie Ngo: Collection and/or assembly of data, data analysis and interpretation. Hsiao-Tzu Ni: Conception and design, collection and/or assembly of data, data analysis and interpretation, final approval of manuscript.

\section{References}

1. Caplan A (1991) Mesenchymal stem cells. J Orthop Res 5: 641-650.

2. Haynesworth SE, Baber MA, Caplan A (1992) Cell surface antigens on human marrow-derived mesenchymal cells are detected by monoclonal antibodies. Bone 13: 69-80.

3. Pittenger MF, Mackay AM, Beck SC, Jaiswal RK, Douglas R, et al. (1999) Multilineage potential of adult human mesenchymal stem cells. Science 284: 143-147.

4. Horwitz EM, Dominici M (2008) How do mesenchymal stromal cells exert their therapeutic benefit? Cytotherapy 10: 771-774.

5. Bianco P, Robey PG, Simmons PJ (2008) Mesenchymal stem cells: Revisiting history, concepts, and assays. Cell Stem Cell 2: 313-319.

6. Friedenstein AJ, Petrakova KV, Kurolesova A, Frolova GP (1968) Heterotopic of bone marrow. Analysis of precursor cells for osteogenic and hematopoietic tissues. Transplantation 2: 230-247.

7. Zuk PA, Zhu M, Mizuno H, Huang J, Futrell JW, et al. (2001) Multilineage cells from human adipose tissue: Implications for cell-based therapies. Tissue Eng 2: 211-228.

8. Gronthos S, Mankani M, Brahim J, Huang J, Futrel JW, et al. (2000) Postnatal human dental pulp stem cells (DPSCs) in vitro and in vivo. Proc Natl Acad Sci USA 97: 13625- 13630.

9. Troyer DL, Weiss ML (2008) Wharton's jelly-derived cells are a primitive stromal cell population. Stem Cells 3: 591-599.

10. Campagnoli C, Roberts IA, Kumar S, Bennett PR, Bellantuono I, et al. (2001) Identification of mesenchymal stem/progenitor cells in human first-trimester fetal blood, liver, and bone marrow. Blood 98: 2396-2402.

11. Fukuchi $Y$, Nakajima $H$, Sugiyama D, Sugiyama D, Hirose I, et al. (2004) Human placenta-derived cells have mesenchymal stem/ progenitor cell potential. Stem Cells 22: 649-658.

12. Tsai MS, Lee JL, Chang YJ, Chang YJ, Hwang SM (2004) Isolation of human multipotent mesenchymal stem cells from secondtrimester amniotic fluid using a novel two-stage culture protocol. Hum Reprod 19: 1450-1456.

13. Horwitz EM, Le Blanc K, Dominici MM, Mueller I, SlaperCortenbach I, et al. (2005) Clarification of the nomenclature for MSC: The International Society for Cellular Therapy position statement. Cytotherapy 7: 393-395.

14. Strioga M, Viswanathan S, Darinskas A, Slaby O, Michalek J (2012) Same or not the same? Comparison of adipose tissuederived versus bone marrow-derived mesenchymal stem and stromal cells. Stem Cells Dev 21: 2724-2752.

15. Yamahara K, Harada K, Ohshima M, Ishikane S, Ohnishi S, et al. (2014)Comparison of angiogenic, cytoprotective, and 
immunosuppressive properties of human amnion- and chorionderived mesenchymal stem cells. PLoS One 9: e88319.

16. Collins E, Gu F, Qi M, Molano I, Ruiz P, et al. (2014) Differential efficacy of human mesenchymal stem cells based on source of origin. J Immunol 193: 4381-4390.

17. New SE, Alvarez-Gonzalez C, Vagaska B, Gomez SG, Bulstrode NW, et al. (2015) A matter of identity-phenotype and differentiation potential of human somatic stem cells. Stem Cell Res 15: 1-13.

18. Dai L, Hu X, Zhang X, Zhu J, Zhang J, et al. (2015) Different tenogenic differentiation capacities of different mesenchymal stem cells in the presence of BMP-12. J Transl Med 13: 200.

19. Dasari VR, Veeravalli KK, Dinh DH (2014) Mesenchymal stem cells in the treatment of spinal cord injuries: A review. World J Stem Cells 6: 120-133.

20. Bailey AM, Mendicino M, Au P (2014) An FDA perspective on preclinical development of cell-based regenerative medicine products. Nat Biotechnol 32: 721-3.

21. Hemeda H, Giebel B, Wagner W (2014) Evaluation of human platelet lysate versus fetal bovine serum for culture of mesenchymal stromal cells. Cytotherapy 2: 170-180.

22. Sundin M, Ringden $O$, Sundberg B, Nava S, Gotherstrom C, et al. (2007) No alloantibodies against mesenchymal stromal cells, but presence of anti-fetal calf serum antibodies, after transplantation in allogeneic hematopoietic stem cell recipients. Haematologica 9: 1208-1215.

23. Gottipamula S, Mittig MS, Kolkundkar U, Seetharam RN (2013) Serum-free media for the production of human mesenchymal stromal cells: A review. Cell Prolif. 46: 608-627.

24. Brunner D, Frank J, Appl H, Schoffl H, Pfaller W, et al. (2010) Serum-free cell culture: The serum-free media interactive online database. ALTEX 1: 53-62.

25. Dromard C, Bourin P, Andre M, De Barros S, Casteilla L, et al. (2011) Human adipose derived stroma/stem cells grow in serum-free medium as floating spheres. Exp Cell Res 6: 770-780.

26. Mark P, Kleinsorge M, Gaebel R, Lux CA, Toel A, et al. (2013) Human mesenchymal stem cells display reduced expression of CD105 after culture in serum-free medium. Stem Cells Intl 2013: 1-8.

27. Patriskoski M, Juntunen M, Boucher S, Campbell A, Vemuri MC, et al. (2013) Development of fully defined xeno-free culture system for the preparation and propagation of cell therapycompliant human adipose stem cells. Stem Cell Res. 4: 1-15.
28. Swamynathan $P$, Venugopal $P$, Kannan $S$, Thej $C$, Kolkundar U, et al. (2014) Are Serum- free and xeno-free culture conditions ideal for large scale clinical grade expansion of Wharton's jelly derived mesenchymal stem cells? A comparative study. Stem Cell Res 5: 1-17.

29. Al-Saqi HS, Saliem M, Asikainen S, Quezada HC, Ekblad A, et al. (2014) Defined serum- free media for in vitro expansion of adipose-derived mesenchymal stem cells. Cytotherapy 16: 915-926.

30. Oikonomopoulos A, Van Deen WK, Manansala AR, Lacey PN, Tomakili TA, et al. (2015) Optimization of human mesenchymal stem cell manufacturing: The effects of animal/xeno-free media. Sci Rep 5: 16570.

31. Pal R, Hanwate M, Jan M, Totey S (2009) Phenotypic and functional comparison of optimum culture conditions for upscaling of bone marrow-derived mesenchymal stem cells. J Tissue Eng Regen Med 3: 163-174.

32. Gottipamula S, Ashwin KM, Muttigi MS, Kannan S, Kolkundkar U, et al. (2014) Isolation, expansion and characterization of bone marrow-derived mesenchymal stromal cells in serum-free conditions. Cell Tissue Res 356: 123-135.

33. Bakopoulou A, Apatzidou D, Aggelidou E, Gousopoulou E, Leyhausen G, et al. (2017) Isolation and prolonged expansion of oral mesenchymal stem cells under clinical-grade, GMPcompliant conditions differentially affects "stemness" properties. Stem Cell Res Ther 8: 247.

34. Bobis-Wozowicz S, Kmiotek K, Kania K, Karnas E, LabedzMaslowska A, et al. (2017) Diverse impact of xeno-free conditions on biological and regenerative properties of hUCMSCs and their extracellular vesicles J Mol Med (Berl) 95: 205-220.

35. Chase LG, Yang S, Zachar V, Yang Z, Lakshmipathy U, et al. (2012) Development and characterization of a clinically compliant xeno-free culture medium in good manufacturing practice for human multipotent mesenchymal stem cells. Stem Cells Transl Med 1: 750-758.

36. Simoes IN, Boura JS, Dos Santos F, Andrade PZ, Cardoso CMP, et al. (2013) Human mesenchymal stem cells from the umbilical cord matrix: Successful isolation and ex- vivo expansion using serum-/xeno-free culture media. Biotechnol J 8: 448-458.

37. Sanchez-Elsner T, Botella LM, Velasco B, Langa C, Bernabeu C, et al. (2002) Endoglin expression is regulated by transcriptional cooperation between the hypoxia and transforming growth factor-pathways. J Biol Chem 46: 43799-43808. 